Int. J. Dev. Biol. 51: 79-84 (2007)

doi: $10.1387 / \mathrm{ijdb} .062204 \mathrm{~cd}$

Short Communication

\title{
A change in response to Bmp signalling precedes ectodermal fate choice
}

\author{
CHRIS T. DEE\#, ABIGAIL GIBSON\#,1 ANDREA RENGIFO\#, SHUN-KUO SUN, ROGER K. PATIENT² \\ and PAUL J. SCOTTING* \\ Institute of Genetics, University of Nottingham, OMC, Nottingham, UK
}

\begin{abstract}
Bone morphogenetic protein (Bmp) signalling plays a central role in the decision of ectoderm to adopt either neural or non-neural fates. The effects of this signalling are seen at midgastrulation in the activation of genes such as the Gata factors and the repression of genes such as the SoxB1 transcription factors in the non-neural regions. Using zebrafish embryos, we show that this Bmp signalling does not repress the expression of these same neural markers just 2-3 hours earlier. Since expression of the Bmp signalling effector, Smad1, only begins during early gastrulation, we tested the role of Smad1 and Smad5 (which is maternally expressed) in controlling gene expression both before and during gastrulation. This showed that the absence of Smad1 does not explain the lack of response of neural genes to Bmp signalling at early stages. However, these experiments showed that expression of the non-neural marker, gata2, is mediated by Smad5 in the absence of Smad1 at early stages, but is dependent upon Smad1 at later stages. Hence, we have shown a dynamic change in the molecular machinery underlying the Bmp response in the ectoderm during gastrulation stages of development.
\end{abstract}

KEY WORDS: ectoderm, neural induction, Bmp, Smad, zebrafish

During vertebrate development the nervous system forms as a results of the ectoderm becoming divided into regions fated to become neural or non-neural (primarily epidermal) tissue. Current models for this process include a central role for Bmps in inhibiting neural fate such that cells only adopt a neural fate where Bmp signalling is blocked or absent (Weinstein and HemmatiBrivanlou, 1999; Munoz-Sanjuan and Brivanlou, 2002). This is achieved on the dorsal side of the embryo by the production of Bmp antagonists, such as Noggin, Follistatin and Chordin (Weinstein and Hemmati-Brivanlou, 1999; Munoz-Sanjuan and Brivanlou, 2002), which physically interact with Bmp ligands to prevent activation of their receptors. This simple model in which the ectoderm adopts a neural fate unless instructed to a nonneural fate by Bmp is referred to as the 'neural default model'. However, Fgf signals have also been shown to be an important factor in the acquisition of neural fate, with a predominant role in posterior regions (Storey etal., 1998; Kudoh etal., 2004, Rentzsch et al., 2004). Fgf signals have been suggested to have a neural inducing function independent of Bmp signalling, but have also been shown to be capable of blocking Bmp signalling by inhibiting the intracellular effector, Smad1 (Pera et al., 2003, Sater et al.,
2003). Also, work in chick and Xenopus laevishas shown that the earliest phase of neural induction occurs prior to gastrulation (Streit et al., 2000) and that inhibition of Bmp signalling is only required as a later step in the selection of neural fate (Linker and Stern, 2004; Stern, 2005). Thus, it is increasingly clear that intercellular signals during early stages of development are likely to establish a 'pre-neural' state in the ectoderm prior to the time at which the cells of the ectoderm make a choice between neural and non-neural fates. However, Bmp signalling and its inhibition, both via soluble antagonists and at an intracellular level by the Fgf signalling pathway, still appears to play a central role in the final definition of neural and non-neural territories of the ectoderm.

In the zebrafish, Danio rerio, the domains of prospective neural and non-neural ectoderm have been defined by fate mapping (Schier and Talbot, 2005) and can be clearly visualized at midgastrulation by the expression of specific genes. In particular, several members of the soxB1 family, such as sox2and sox3, are

Abbreviations used in this paper: Bmp, bone morphogenic protein; Fgf, fibroblast growth factor; $\operatorname{nog} 1 \& 2$, noggin 1 and noggin 2; caBmpR, constitutively active Bmp receptor; MO, morpholino.

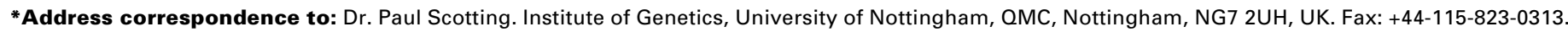
e-mail: Paul.Scotting@nottingham.ac.uk

* Note: The indicated authors contributed equally to this study.

1 Current address: Victor Chang Cardiac Research Institute, 384 Victoria Street, Darlinghurst NSW 2010, Sydney, Australia.

2 Current address: Weatherall Institute of Molecular Medicine, University of Oxford, John Radcliffe Hospital, Headington, Oxford, OX3 9DS, UK.
} 


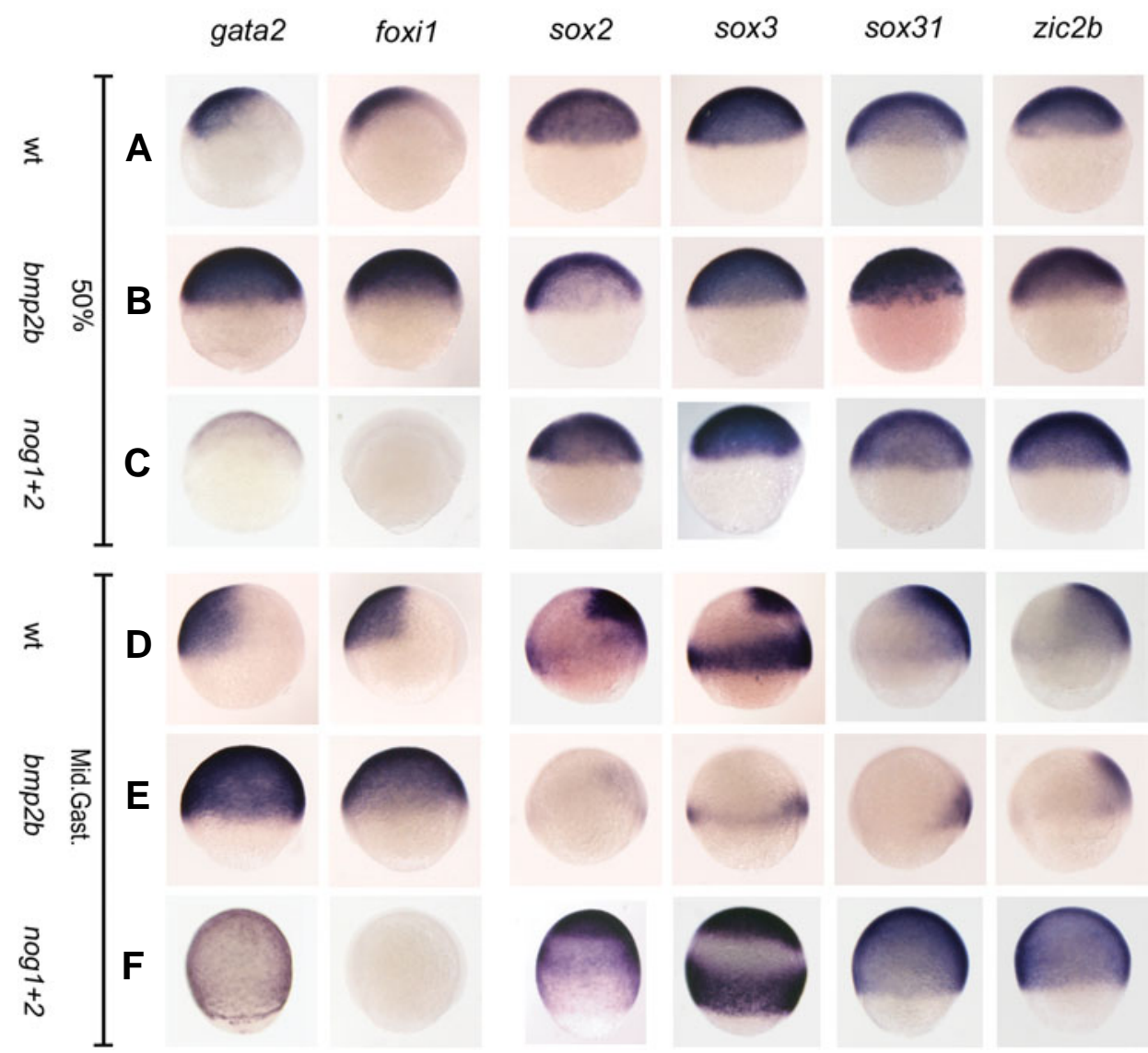

Fig. 1. Early markers of neural fate are insensitive to Bmp signalling at $\mathbf{5 0} \%$ epiboly. Lateral views of embryos with dorsal to the right. The effect of manipulating Bmp signalling was compared at the $50 \%$ epiboly (A-C) and mid-gastrula (D-F) stages. Prospective non-neural fate marker genes (gata2, foxi1) and prospective neural fate marker genes (sox2, sox3, sox31 and zic2b) analysed are indicated at the top of panels. Injection of bmp2b mRNA caused expansion of gata2 and foxi1 expression at $50 \%$ epiboly, but had no effect on sox 2 , sox3, sox31 or zic $2 \mathrm{~b}$ (B) as compared to control embryos (A). Injection of $\mathrm{bmp} 2 \mathrm{~b} m R N A$ resulted in expansion of gata2 and foxi1 and repression of sox2, sox3, sox31 and zic2b in mid-gastrula embryos (E) compared to controls (D). Injection of nog $1+2$ mRNA caused loss of gata2 and foxi1 at both 50\% epiboly and mid-gastrulation stages, but had no effect on sox2, sox3, sox31 or zic $2 \mathrm{~b}$ at $50 \%$ epiboly (C), whilst resulting in expansion of all prospective neural markers in mid-gastrula embryos (F).

among the first transcription factors to be expressed in a spatial and temporal pattern consistent with the acquisition of neural fate in the ectoderm (Fig. 1D) (Okuda et al., 2006). Similarly, genes such as gata2 and foxi1 have been shown to be restricted to ectodermal cells fated to lie outside the CNS (Fig. 1D) (Solomon et al., 2003; Rentzsch et al., 2004; Londin et al., 2005). Importantly, the expression of these markers has recently been shown to be imposed by a precisely regulated combination of Bmp and Fgf signals (Kudoh et al., 2004, Rentzsch et al., 2004) and these observations are supported by our own data (see Figs. 1,2). Ectopically increasing Bmp signalling leads to the expansion of prospective non-neural ectoderm at the expense of prospective neural ectoderm (Kudoh et al., 2004, Rentzsch et al., 2004; Delaune et al., 2005). However, despite having distinct, mutually exclusive patterns of expression by mid-gastrulation stages (70$80 \%$ epiboly, see Fig. 1D), we noted that at the onset of gastrulation, or immediately prior to it (up to $50 \%$ epiboly), various markers of neural fate, including sox2 and sox3, are expressed throughout the ectoderm and are consequently co-expressed with markers of nonneural fate, such as gata2 and foxi1 (Fig. 1A). This observation suggested a potentially significant change in the regulation of these markers between the stages of $50 \%$ and $70 \%$ epiboly. We therefore set out to examine the control of these markers at the earlier stage, an analysis that revealed a striking difference in the response of the ectoderm to Bmp and Fgf signalling at these two stages.

Injection of mRNA encoding the Bmp2b ligand resulted in expansion of the nonneural markers gata2 and foxi 1 into dorsal regions of the ectoderm normally fated to form neural tissue (Fig. 1E) and a reciprocal repression in the dorsoanimal domain of neural markers such as sox2, sox3, sox31 and zic2b (Fig. 1E) at the mid-gastrulation (70\%-90\% epiboly) stage. In addition, blocking Bmp signalling by injecting mRNA encoding the Bmp antagonists Noggin1 and Noggin2 (Nog1\&2) (Furthauer et al., 1999) was sufficient to cause loss of gata2and foxi1 expression, whilst the expression domain of markers of the prospective neural ectoderm expanded into the ventral region normally fated to lie outside the CNS (Fig. $1 \mathrm{~F})$. However, analysis of embryos at $50 \%$ epiboly following injection of a dose of bmp2b mRNA that was sufficient to repress prospective neural markers at mid-gastrulation (Fig. 1E) showed no discernable effect on the expression of sox2, sox3, sox31 or zic2b (Fig. 1B), either in terms of pattern or overall strength of expression. Likewise, these same genes were unaffected at $50 \%$ epiboly following injection of nog $1 \& 2$ mRNA to block Bmp signaling (Fig. 1C). Importantly, all of the above treatments did affect gata2 and foxi1 (Fig. 1 B,C). It is interesting to note that at this earlier stage, gata2 and foxi1 are already expressed in a ventrally restricted pattern that appears to anticipate their later expression restricted to the non-neural ectoderm. The effect of injection of bmp2bor nog1\&2mRNA on the expression of non-neural genes demonstrates that the Bmp pathway is active at $50 \%$ epiboly, but unlike the response at mid-gastrulation (only 2-3 hours later), neural markers are unaffected.

We next examined the effect of Fgf signals at the $50 \%$ epiboly stage. Fgf signals have a neural inducing role in the zebrafish ectoderm and are able to make cells unresponsive to Bmp signalling at mid-gastrulation (Kudoh et al., 2004; Rentzsch et al., 2004). We therefore tested whether the lack of response of some genes to Bmp signalling at $50 \%$ epiboly was due to intracellular inhibition of the Bmp pathway by Fgf signalling. If this were the case, blocking Fgf signalling would relieve this inhibition and make neural markers sensitive to endogenous Bmp signals and 


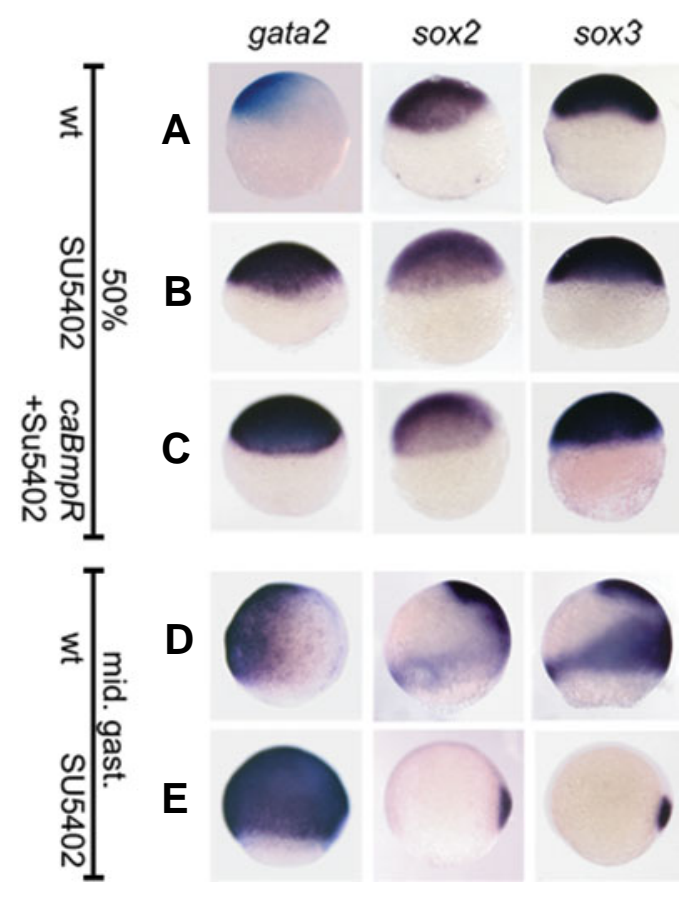

Fig. 2. Early markers of neural fate are insensitive to Fgf signalling at $\mathbf{5 0 \%}$ epiboly. Lateral views of embryos with dorsal to the right. Blocking Fgf signalling with SU5402 caused expansion of gata2 expression at 50\% epiboly, but had no effect on sox2 or sox3 (B) as compared to control embryos (A). SU5402 caused expansion of gata2 and repression of sox2 and sox3 in mid-gastrula embryos (E) compared to controls (D). Injection of caBmpR mRNA with SU5402 treatment caused expansion of gata2, but again had no effect on sox2 or sox3 expression at 50\% epiboly (C).
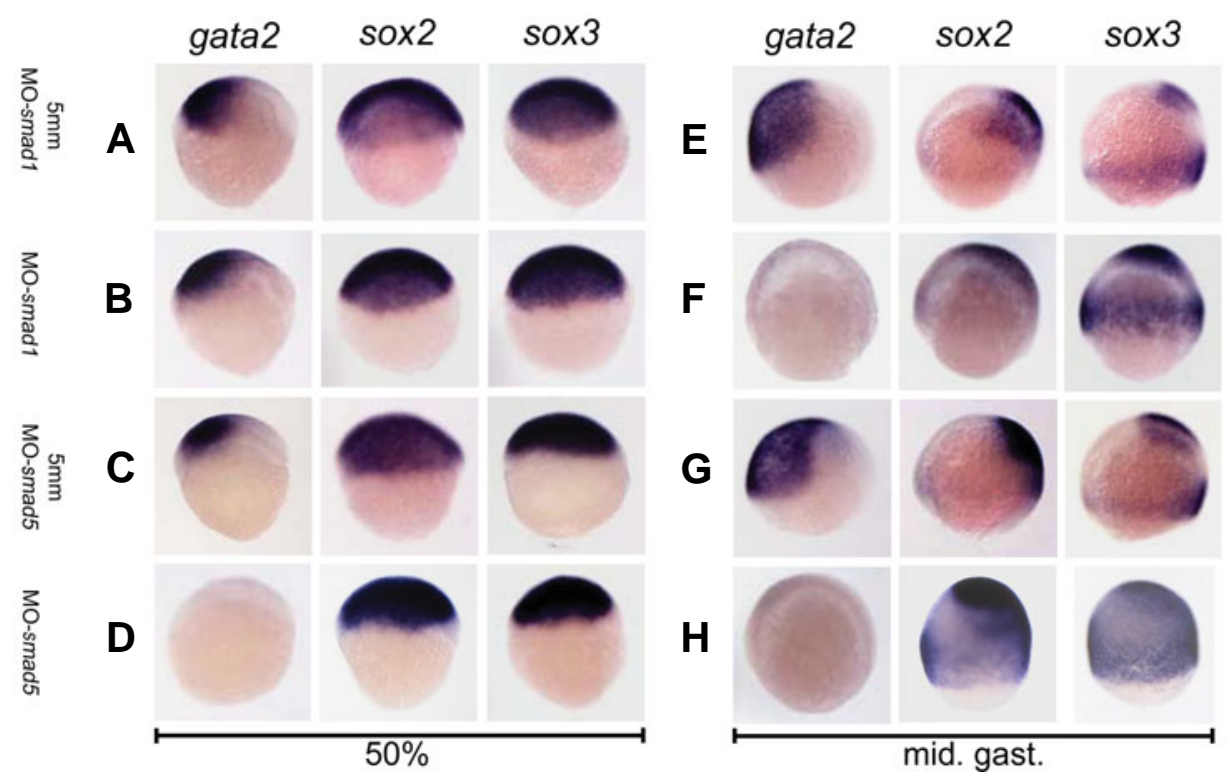

Fig. 3. The effect of smad1 and smad5 morpholino knockdown on early markers of ectodermal fate. Lateral views with dorsal to the right. Injection of $5 \mathrm{mmMO}$-smad1 (A,E) or $5 \mathrm{mmMO}$-smad5 $(\mathbf{C}, \mathbf{G})$ controls did not affect expression of gata2, sox2 or sox3. Injection of $\mathrm{MO}$-smad1 did not affect gata2, sox2 or sox 3 expression at $50 \%$ epiboly (B), but caused loss of gata2 and expansion of sox 2 and sox3 expression in mid-gastrula embryos (F). Injection of $\mathrm{MO}$-smad5 caused loss of gata2 expression at $50 \%$ epiboly, but had no effect on sox 2 or sox3 expression at this stage (D). MO-smad5 resulted in loss of gata2 and expansion of sox2 and sox3 at mid-gastrulation (H). so result in the loss of sox2 and sox3. However, although treatment of embryos with SU5402 did cause gata2expression to expand throughout the ectoderm at $50 \%$ epiboly (Fig. 2B) and caused almost complete loss of both sox2 and sox3 at midgastrulation (Fig. 2E), it did not alter the expression of either sox2 or sox3at $50 \%$ epiboly (Fig. 2B). In order to ensure that this lack of effect at $50 \%$ epiboly was not due to insufficient endogenous Bmp being present, we repeated the above experiment treating with SU5402 after first injecting the embryos with mRNA encoding a constitutively active form of Bmp-receptor (caBmpR). Once again, sox2and sox3expression was unaltered (Fig. $2 \mathrm{C}$ ) despite strong activation of gata2. Similarly, injection of mRNA encoding eFgf was sufficient for complete repression of gata2 at $50 \%$ epiboly, but had no detectable effect on sox2 or sox3, despite causing expansion of neural fate at mid-gastrulation (data not presented). Thus, it appears that there is a fundamental change in Bmp responsiveness of the ectoderm between $50 \%$ epiboly and mid-gastrulation which is likely to reflect some change in the signalling machinery of ectodermal cells downstream of the Bmp receptors.

Intracellular transduction of Bmp signalling in zebrafish is primarily mediated by the transcription factors Smad1 and Smad5 (Dick et al., 1999). It is clear, however, that Smad1 and Smad5 play distinct roles in the development of ventral fates. Smad5 is present in the earliest stages of development, initially as a maternal factor and analysis of mutants and morphants has shown it to be essential for normal development of ventrally specified fates such as epidermis. smad5loss-of-function results in loss of gata2 in the mid-gastrula ectoderm and causes severe defects in the later development of ventral tissues similar to the bmp mutants swirl (bmp2b) and snailhouse (bmpt) (Dick et al., 1999; Hild et al., 1999). Importantly, Smad5-mediated Bmp signalling is essential for expression of smad1, which is undetectable at the earliest stages of development and is only initially detected at very low levels from $50 \%$ epiboly. smad1 expression increases substantially by mid-gastrulation when the protein is thought to play a major role in the regulation of genes controlling ventral fates, such as non-neural fate in the ectoderm (Dick et al., 1999; Hild et al., 1999). However, the relative importance of Smad5 and Smad1 in regulating Bmpresponsive genes at later stages, when both are present, has not been directly tested. The appearance of Smad1 coincides with the time at which the neural markers become sensitive to Bmp signalling. Thus, the change in Smad1 expression could explain this change in Bmp-sensitivity.

In order to examine the respective roles of Smad1 and Smad5 at the 50\% epiboly and mid-gastrula stages, we designed morpholino anti-sense oligonucleotides (MOs) targeted to specifically block the translation of smad1 or smad5 tran- 
scripts, designated MO-smad1 and MO-smad5. In addition, we designed 5-base pair mismatch MOs (5mmMO-smad1 and $5 \mathrm{mmMO}$-smad5) as negative controls. Although the consequences of smad5 loss-of-function have previously been examined by analysis of mutants and anti-sense morpholino knockdowns (Hild et al., 1999; Lele et al., 2001; Kramer et al., 2002), the effects of specific loss of smad1 have yet to be reported. Both MO-smad1 and $\mathrm{MO}$-smad5resulted in a strong dorsalization phenotype at 24 hours similar to that seen in bmpmutants and in embryos treated with bmpmorpholinos (Lele et al., 2001) (data not presented). In line with the role of Smad5 downstream of Bmp signalling, injection of MO-smad5 was sufficient to cause complete loss of the non-neural ectoderm marker gata2 at the $50 \%$ epiboly stage (Fig. 3D). Morpholino knockdown of smad1, which is only expressed at low levels at $50 \%$ epiboly, had no effect on the expression of gata2(Fig. 3B). Consistent with the above data in which Bmp signalling was manipulated, neither morpholino caused any effect on the expression of sox2 or sox3 at this stage (Fig. 3 B,D). Therefore, at $50 \%$ epiboly, Smad5 is essential for the regulation of gata2and is able to perform this function in a manner independent of Smad1. By contrast, at mid-gastrulation injection of either MO-smad5 or MO-smad1 resulted in complete loss of gata2expression (Fig. $3 \mathrm{~F}, \mathrm{H}$ ). In addition, markers of prospective neural fate, sox2 and sox3, were found to be expanded towards the ventral side of the embryo (Fig. $3 \mathrm{~F}, \mathrm{H}$ ). This demonstrates an absolute requirement for $\mathrm{Smad} 1$ in regulating $\mathrm{Bmp}$ target genes at this stage of development, in contrast to the situation at $50 \%$ epiboly. Critically, at mid-gastrulation, endogenous Smad5 cannot compensate for the loss of Smad1, despite being able to fulfill this function independently during earlier development. This implies that a fundamental change in the molecular machinery that converts instructive Bmp signals into a choice of cell fate occurs in the period following the onset of gastrulation (Fig. 4). In all cases, injection of the control morpholinos $5 \mathrm{mmMO}$-smad1 and $5 \mathrm{mmMO}$-smad5had no effect on the expression of ectodermal genes (Fig. 3A, C, E and G).

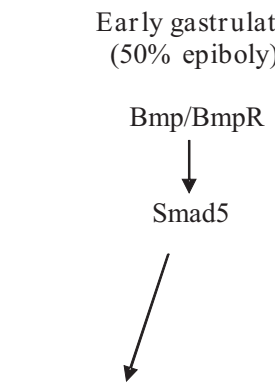

non-neural genes neural genes
Mid-gastrulation (70-90\% epiboly)

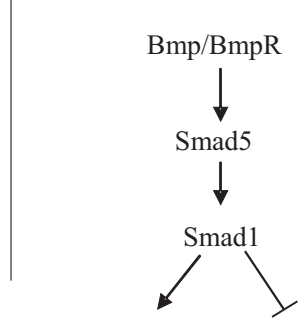

non-neural genes neural genes
Fig. 4. Model for Bmp signalling during zebrafish gastrulation. This model reflects a change in the Bmp machinery in which early Bmp signalling acts through Smad5, while later signalling makes use of Smad1. Thus, during early gastrulation, non-neural genes are dependent upon signalling via Smad5, whereas genes that are later restricted to neural ectoderm are unaffected by Smad5 and are therefore expressed regardless of Bmp signalling. By late gastrulation, smad1 expression is activated in response to Smad5, and Smad1 is now required for expression of non-neural genes and repression of neural genes by Bmp signalling. It should be noted that our data do not exclude a role for Smad5 in directly regulating gene expression during gastrulation.
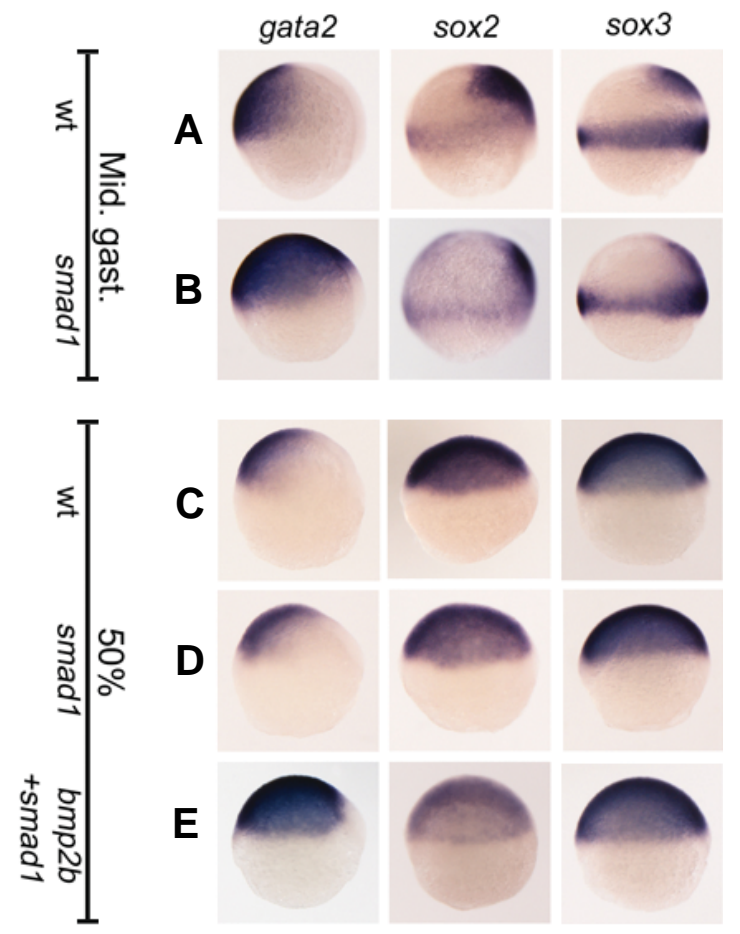

Fig. 5. Smad1 cannot regulate marker gene expression at $\mathbf{5 0} \%$ epiboly. Lateral views with dorsal to the right. Injection of smad1 mRNA caused expansion of gata2 and repression of sox2 and sox3 in midgastrula embryos (B) as compared to wild-type controls (A). Exogenous smad1 had no effect on the expression of any of these markers at $50 \%$ epiboly (D) as compared to control embryos (C). Co-injection of smad1 $m R N A$ with bmp2b mRNA resulted in expansion of gata2, but had no effect on sox3 and only a weak repressive effect on sox2 (E).

Since sox 2 and sox 3 expression is only affected by Bmp signalling once endogenous smad1 expression appears, we next asked if ectopic expression of smad1 prior to gastrulation would lead to repression of sox2and sox3expression at the 50\% epiboly stage. We injected embryos with smad1mRNA and compared the responses at $50 \%$ epiboly to mid-gastrulation (when smad1 is essential for normal patterning of the ectoderm (Fig. 3). At midgastrulation, ectopic smad1 (or smad5, data not shown) had a similar effect to over-expression of the Bmp2b ligand, causing expansion of non-neural ectoderm marked by gata2(as has been described previously, Kramer et al., 2002) and reciprocal reduction in the domain expressing the neural ectoderm markers, sox2 and sox3(Fig. 5B). At $50 \%$ epiboly, expression of sox2and sox3 was unaffected by exogenous smad1 (even at a very high dose of 350pg) (Fig. 5D). More surprisingly, little effect was seen on the expression of gata2 at this stage (Fig. 5D) in contrast to the expansion of gata2expression seen following over-expression of upstream Bmp (Fig. 1B). smad5 RNA did expand expression of gata2but had no effect on sox3expression, just as was seen with bmp2b injections (data not shown). Although overexpression of unphosphorylated Smads can cause effects that mimic Bmp signalling (Dick et al., 1999) this effect might be weak compared to the effect that activated (phosphorylated) Smads would have. We therefore tested the effect of co-injecting either bmp2b (Fig. $5 E)$ mRNA along with the smad1 mRNA so that the exogenous Smad1 should be phospho-activated by the Bmp pathway through- 
out the ectoderm. In this case, expression of gata2 was expanded, as it was when Bmp2b (see Fig. 1) was over-expressed independently. Similar results were obtained following injection with caBmpRalone or in combination with smad1RNA (data not presented). However, expression of sox 3 remained completely unaffected by these treatments (Fig. 5E). A weak repressive effect could be observed on the overall level of sox2 expression (Fig. 5E), although the domain of expression was not restricted in the manner observed at mid-gastrula stages suggesting that it might represent an artifact of driving the Bmp pathway to excess.

This study describes a new phenomenon central to the ability of ectoderm to undergo the fate choice between neural and nonneural ectoderm through the process of neural induction. Our data suggest that there is a fundamental change in the Bmp response machinery between early gastrulation (50\% epiboly) and midgastrulation. Prior to this change certain 'neural' genes are not responsive to Bmp signalling, but become responsive at the later stage. Since these genes are transcription factors implicated in mediating fate choice as part of neural induction, this change in responsiveness is likely to be a key event in priming the cells for neural induction to take place. Although the appearance of smad1 mRNA at this same stage provided a strong candidate for the necessary change in Bmp responsive machinery, our data show that absence of smad1 at the earlier stage is not the explanation for the differing response to Bmp signalling. Thus, the molecular explanation for this change remains to be determined. Our data also provide insight into the dynamic changes in the role of Smad1 and Smad5 in the ectoderm during this early period of development. At early stages, gata2 is controlled via Smad5 (completely independently of Smad1), while at the later stage, its expression appears to be entirely dependent upon Smad1 (Fig. 4). Thus, our study shows that Bmp signalling in the ectoderm is a highly dynamic process with the detail of regulation of individual genes changing rapidly with time.

\section{Experimental Procedures}

\section{Preparation and injection of $m R N A$, injection of morpholinos and SU5402 treatment}

Capped mRNAs for bmp2b(Kudoh et al., 2004), noggin1 and noggin2 (Furthauer et al., 1999), constitutively active BmpR (caBmpR) (Nikaido et al., 1997), smad1 (Dick et al., 1999) and smad5 (Hild et al., 1999) were produced from linearised cDNA templates using mMessage-Machine Kits (Ambion, US) according to the manufacturers instructions. The following quantities of mRNAs were injected in a $0.5 \mathrm{nl}$ volume at the 1-4 cell stage: bmp2b (50pg), noggin 1+ noggin2(10pg each), caBmpR(150350pg), efgf (10pg), smad1 (350pg), smad5 (325pg). In each experiment, all embryos were injected with identical quantities of a particular mRNA, allowing the responses at different stages of development to be compared directly.

Morpholino antisense oligonucleotides (Gene Tools, US) were: MOsmad1 (5'-AGGAAAAGAGTGAGGTGACATTCAT-3'), MO-smad5 (5'AAAACAGACTAGACATGGAGGTCAT-3'), 5mmMO-smad1 (5'AGcAAAAcAGTGAcGTGAgATTgAT-3'), 5mmMO-smad5 (5'AAAAgAGAgTAGAgATGGAcGTgA T-3'). All morpholinos were injected at the $1-2$ cell stage in a $0.5 \mathrm{nl}$ volume at a concentration of $5 \mathrm{ng}$ unless otherwise stated in the text.

SU5402 (Calbiochem; dissolved in DMSO to give $8.437 \mathrm{mM}$ stock solution) was used at a final concentration of $20-50 \mu \mathrm{M}$ in fish water containing methyl blue. Embryos were treated in their chorions at $28^{\circ} \mathrm{C}$, in the absence of light, just prior to MBT until the time of collection. Control embryos were treated with an equivalent dilution of DMSO.

\section{Whole-mount in situ hybridisation}

Whole mount in situ hybridisation on zebrafish embryos was carried out as previously described (Jowett and Yan, 1996). Digoxigenin (DIG)labelled riboprobes were transcribed from linearised templates using T3, T7 or SP6 RNA polymerase (Promega) in the presence of DIG-labelled nucleotides (Roche Ltd., UK). Detection of alkaline phosphatase-conjugated DIG antibody was performed using BM-purple (Roche Ltd., UK). Probes were used as described previously: gata2 (Read et al., 1998), zic2b (Toyama et al., 2004), sox31 (Girard et al., 2001) or obtained as ESTs from RZPD: sox2(GenBank Accession Number: AW280493), sox3 (GenBank Accession Number: Al959362), foxi1 (GenBank Accession Number: CF997841).

\section{Acknowledgements}

We thank Dave Reffin and Elaine Thorpe for invaluable technical assistance. Research was supported by grants from MRC and BBSRC. AG was supported by a University of Nottingham studentship, AR holds a BBSRC studentship.

\section{References}

DELAUNE, E., LEMAIRE, P. and KODJABACHIAN, L. (2005). Neural induction in Xenopus requires early Fgf signalling in addition to Bmp inhibition. Development 132: 299-310.

DICK, A., MEIER, A. and HAMMERSCHMIDT, M. (1999). Smad1 and Smad5 have distinct roles during dorsoventral patterning of the zebrafish embryo. Dev Dyn 216: $285-98$.

FURTHAUER, M., THISSE, B. and THISSE, C. (1999). Three different noggin genes antagonize the activity of bone morphogenetic proteins in the zebrafish embryo. Dev Bio/214: 181-96.

GIRARD, F., CREMAZY, F., BERTA, P. and RENUCCI, A. (2001). Expression pattern of the sox31 gene during zebrafish embryonic development. Mech Dev 100: $71-3$.

HILD, M., DICK, A., RAUCH, G.J., MEIER, A., BOUWMEESTER, T., HAFFTER, P. and HAMMERSCHMIDT, M. (1999). The smad5 mutation somitabun blocks Bmp2b signaling during early dorsoventral patterning of the zebrafish embryo. Development 126: 2149-59.

JOWETT, T. and YAN, Y.L. (1996). Double fluorescent in situ hybridization to zebrafish embryos. Trends Genet 12: 387-9.

KRAMER, C., MAYR, T., NOWAK, M., SCHUMACHER, J., RUNKE, G., BAUER, H., WAGNER, D.S., SCHMID, B., IMAI, Y., TALBOT, W.S. et al. (2002). Maternally supplied Smad5 is required for ventral specification in zebrafish embryos prior to zygotic Bmp signaling. Dev Bio/250: 263-79.

KUDOH, T., CONCHA, M.L., HOUART, C., DAWID, I.B. and WILSON, S.W. (2004). Combinatorial Fgf and Bmp signalling patterns the gastrula ectoderm into prospective neural and epidermal domains. Development 131: 3581-92.

LELE, Z., BAKKERS, J. and HAMMERSCHMIDT, M. (2001). Morpholino phenocopies of the swirl, snailhouse, somitabun, minifin, silberblick and pipetail mutations. Genesis 30: 190-4.

LINKER, C. and STERN, C.D. (2004). Neural induction requires Bmp inhibition only as a late step and involves signals other than Fgf and Wnt antagonists. Development 131: 5671-81.

LONDIN, E.R., NIEMIEC, J. and SIROTKIN, H.I. (2005). Chordin, Fgf signaling and mesodermal factors cooperate in zebrafish neural induction. Dev Bio/279: 1-19.

MUNOZ-SANJUAN, I. and BRIVANLOU, A.H. (2002). Neural induction, the default model and embryonic stem cells. Nat Rev Neurosci3: 271-80.

NIKAIDO, M., TADA, M., SAJI, T. and UENO, N. (1997). Conservation of Bmp signaling in zebrafish mesoderm patterning. Mech Dev61: 75-88.

OKUDA, Y., YODA, H., UCHIKAWA, M., FURUTANI-SEIKI, M., TAKEDA, H., $\mathrm{KONDOH}, \mathrm{H}$. and KAMACHI, Y. (2006). Comparative genomic and expression analysis of group B1 sox genes in zebrafish indicates their diversification during 
vertebrate evolution. Dev Dyn 235: 811-25.

PERA, E.M., IKEDA, A., EIVERS, E. and DE ROBERTIS, E.M. (2003). Integration of Igf, Fgf and anti-Bmp signals via Smad1 phosphorylation in neural induction. Genes Dev 17: 3023-8.

READ, E.M., RODAWAY, A.R., NEAVE, B., BRANDON, N., HOLDER, N., PATIENT, R.K. and WALMSLEY, M.E. (1998). Evidence for non-axial A/P patterning in the nonneural ectoderm of Xenopus and zebrafish pregastrula embryos. Int J Dev Bio/42: 763-74.

RENTZSCH, F., BAKKERS, J., KRAMER, C. and HAMMERSCHMIDT, M. (2004). Fgf signaling induces posterior neuroectoderm independently of Bmp signaling inhibition. Dev Dyn 231: 750-7.

SATER, A.K., EL-HODIRI, H.M., GOSWAMI, M., ALEXANDER, T.B., AL-SHEIKH, O., ETKIN, L.D. and AKIF UZMAN, J. (2003). Evidence for antagonism of Bmp4 signals by Map kinase during Xenopus axis determination and neural specification. Differentiation 71: 434-44.

SCHIER, A.F. and TALBOT, W.S. (2005). Molecular genetics of axis formation in zebrafish. Annu Rev Genet 39: 561-613.

SOLOMON, K.S., KUDOH, T., DAWID, I.B. and FRITZ, A. (2003). Zebrafish foxi1 mediates otic placode formation and jaw development. Development130: 929-40.
STERN, C.D. (2005). Neural induction: Old problem, new findings, yet more questions. Development 132: 2007-21.

STOREY, K.G., GORIELY, A., SARGENT, C.M., BROWN, J.M., BURNS, H.D., ABUD, H.M. and HEATH, J.K. (1998). Early posterior neural tissue is induced by Fgf in the chick embryo. Development 125: 473-84.

STREIT, A., BERLINER, A.J., PAPANAYOTOU, C., SIRULNIK, A. and STERN C.D. (2000). Initiation of neural induction by Fgf signalling before gastrulation. Nature 406: 74-8.

TOYAMA, R., GOMEZ, D.M., MANA, M.D. and DAWID, I.B. (2004). Sequence relationships and expression patterns of zebrafish zic2 and zic5 genes. Gene Expr Patterns 4: 345-50.

WEINSTEIN, D.C. and HEMMATI-BRIVANLOU, A. (1999). Neural induction. Annu Rev Cell Dev Bio/ 15: 411-33.

Received: 28th July 2006 Reviewed by Referees: 30th August 2006 Modified by Authors and Accepted for Publication: 26th September 2006 Published Online: 6th October 2006 